\title{
Impacted Anterior Urethral Calculus Complicated by a Stone-containing Diverticulum in an Elderly Man: Outcome of Transurethral Lithotripsy without Resection of the Diverticulum
}

Tie Zhou, Guanghua Chen, Wei Zhang, Yonghan Peng, Liang Xiao, Chuangliang Xu, Yinghao Sun

Department of Urology, Changhai Hospital, The Second Military Medical University, Shanghai, P. R. China

\section{ABSTRACT}

Introduction: The prevalence of lower urinary tract symptoms (LUTS) is about 20\% in men aged 40 or above. Other than benign prostatic hyperplasia (BPH), urethral diverticulum or calculus is not uncommon for LUTS in men. Surgical treatment is often recommended for urethral diverticulum or calculus, but treatment for an impacted urethral calculus complicated by a stone-containing diverticulum is challenging.

Materials and Methods: An 82-year-old man had the persistence of LUTS despite having undergone transurethral resection of prostate for BPH. Regardless of treatment with broad spectrum antibiotics and an $\alpha$-blocker, LUTS and post-void residual urine volume $(100 \mathrm{~mL})$ did not improve although repeated urinalysis showed reduction of WBCs from 100 to 10 per high power field. Further radiology revealed multiple urethral calculi and the stone configuration suggested the existence of a diverticulum. He was successfully treated without resecting the urethral diverticulum; and a new generation of ultrasound lithotripsy (EMS, Nyon, Switzerland) through a 22F offset rigid Storz nephroscope (Karl Storz, Tuttingen, Germany) was used to fragment the stones.

Results: The operative time was 30 minutes and the stones were cleanly removed. The patient was discharged after 48 hours with no immediate complications and free of LUTS during a 2 years follow-up.

Conclusions: When the diverticulum is the result of a dilatation behind a calculus, removal of the calculus is all that is necessary. Compared with open surgery, ultrasound lithotripsy is less invasive with little harm to urethral mucosa; and more efficient as it absorbs stone fragments while crushing stones.

\section{ARTICLE INFO}

Available at: www.brazjurol.com.br/videos/september_october_2013/Zhou_754_755video.htm

Int Braz J Urol. 2013; 39 (Video \#12): 754-5

Submitted for publication:

May 25, 2013

Accepted after revision:

June 10, 2013
Correspondence address:

Yinghao Sun, M.D.

Department of Urology, Changhai Hospital The Second Military Medical University 168 Changhai Road

Shanghai 200433, P. R. China

Fax: +86 21 3503-0006

E-mail: Yinghao.sun@yahoo.com 


\section{EDITORIAL COMMENT}

The video submitted by Zhou et al. addressing management of an anterior urethral diverticular stone has been revised to the satisfaction of this re- viewer. The addition of narration and other requested details provides a much more informative presentation. It will indeed provide the audience with the details necessary to reproduce the case should one encounter a similarly interesting clinical scenario.

Erik J. Busby, MD

Uro-oncology

Health System

Greenville, South Carolina, USA

E-mail: ebusby@ghs.org 